\title{
Online measurement and evaluation of the Er:YAG laser ablation process using an integrated OCT system
}

\author{
A. Fuchs ${ }^{1}$, M. Schultz ${ }^{2}$ A. Krüger ${ }^{2}$, D. Kundrat ${ }^{1}$, J. Díaz Díaz ${ }^{1}$, T. Ortmaier ${ }^{1}$ \\ ${ }^{1}$ Institute of Mechatronic Systems (Leibniz Universität Hannover), Hannover, Germany, fuchs@imes.uni-hannover.de \\ ${ }^{2}$ Laser Zentrum Hannover e.V., Hannover, Germany
}

\begin{abstract}
Laser surgery has gained clinical importance due to numerous advantages including contact-free processing, arbitrary cutting geometries, and high precision. However, online process control remains a challenge for widespread clinical use. Therefore, we established a combined setup of a pulsed Er:YAG laser $(\lambda=2940 \mathrm{~nm})$ and an optical coherence tomography (OCT) $(\lambda=930 \mathrm{~nm})$ for in situ monitoring of hard tissue ablation. The optical setup facilitates an interactive control of the laser ablation depth and remaining tissue strength through the depth resolution of OCT. The 3D OCT data-set, which is acquired after ablation, provides contours and layer thicknesses.
\end{abstract}

\section{Introduction}

Laser surgery has gained clinical importance especially in soft tissue ablation and ophthalmology due to multiple advantages. They comprise contact-free processing, arbitrary cutting geometries, and high precision [1, 2]. Scannerguided laser ablation also provides an efficient approach to process hard tissue with minor thermal and mechanical trauma in various surgical applications [3]. A precondition for such an advantageous use of lasers is a proper setting of laser parameters which optimize ablation rate and precision while minimizing collateral damage such as coagulation and carbonization [4, 5]. The efficiency of laser tissue interaction and amount of removal is strongly dependent on optical and thermal properties of the target tissue both of which are influenced by the extra- and intracellular water content [6]. Moreover, the ablation rate is governed by the laser properties and settings such as wavelength, focus spot size, pulse duration and energy, and frequency [7]. Up to now, medical hard tissue ablation is restricted due to the missing depth feedback from contactless laser processing. For a more widespread clinical use, in situ measurement and monitoring of ablation geometries is mandatory and an important issue for processing biological tissue.

Optical coherence tomography (OCT) is an attractive candidate for compact, non-invasive, and real time imaging of laser irradiated material [8]. This non-invasive interferometric imaging modality depicts not only a topological profile of examined surfaces but also displays the subsurface structures $[9,10]$. Therefore, OCT is standard technique in ophthalmology for the analysis of retinal diseases [11]. High resolutions are achieved without physical contact and index-matching media. Resolutions of about 10 $\mu \mathrm{m}$ and penetration depths of several millimeters (depending on the optical properties of the sample) allow for distinction between different material layers. OCT combined with surgical lasers has been used for qualitative and quantitative monitoring of the laser ablation process in various soft and hard tissues $[12,13]$.
In our work we suggest an automatic segmentation algorithm for the quantitative OCT based analysis of the ablation site in hard tissue. We present a combined setup of a pulsed Er:YAG laser $^{1} \quad(\lambda=2940 \mathrm{~nm})$ and an OCT $(\lambda=$ $930 \mathrm{~nm})$ for in situ observation and control of hard tissue ablation. We have developed an automatic method based on deformable models for determining depth maps of tissue surfaces captured by OCT. Furthermore, the method is applicable to detect critical anatomical structures and boundaries within the range of the advancing ablation front and to calculate the thickness of the remaining tissue layer. With such quantification, our approach is a step towards image-based control and safe processing, enabling to ablate a predefined depth at specific positions and protecting near-surface as well as underlying anatomical structures in laser surgery.

\section{Methods}

The optical setup facilitates a combined and rigid configuration of OCT and Er:YAG laser. First, the laser beams both pass independent scanners and focussing optics. The optical path of the cutting laser is modelled to fit the requirements of compactness of the whole setup as well as beam and focus quality. Finally, OCT and Er:YAG laser are combined by a custom designed dichroic mirror in order to co-propagate onto the target. Spatial overlap of OCT and addressable focus positions of the cutting laser comprise a volume of $(10 \times 10 \times 10) \mathrm{mm}^{3}$. To determine the spatial relations between components, the Er:YAG scan head origin is registered to the OCT coordinate system by detecting ablation hole centres from single laser pulses.

\subsection{Tissue Ablation}

Our procedure was evaluated by processing and analyzing biological hard tissue samples of a porcine femoral bone.

\footnotetext{
${ }^{1}$ DPM 15 Laser Module (Pantec Engineering/3m.i.k.r.o.n ${ }^{\mathrm{TM}}$ )
} 
These were cut into $20 \mathrm{~mm}$ thick slices while frozen. Thereafter, specimens were thawed to room temperature one hour before laser ablation, and kept moist until processing. Additionally, periosteum, adherent soft tissue, and bone marrow were removed to avoid burning and charring. The inner bone surface was slightly polished with fine grain sandpaper for an improved detection of boundary layers in OCT imaging.

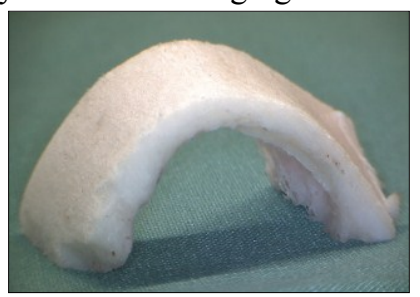

(a)

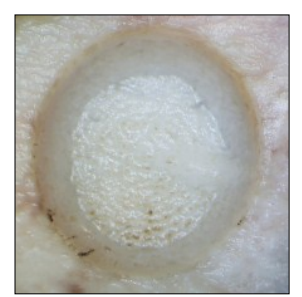

(b)
Image 1: (a) Thawed and cleaned porcine bone specimen. (b) Laser drilled hole ( $8 \mathrm{~mm}$ diameter) after repeated application of circular scan patterns.

The tissue surface was placed within focal distance of the cutting laser. A circular area of $8 \mathrm{~mm}$ diameter was ablated using the laser scanning system. Scan trajectories were composed of concentric circles which are defined as a function of spot diameter, total diameter, and pulse overlap. In consideration of minimal thermal damage, the required laser settings were empirically determined a priori. Pulse duration of $\tau_{p}=150 \mu \mathrm{s}$, repetition rate of $f_{p}=150 \mathrm{~Hz}$, and scanning speed of $v_{s}=20 \mathrm{~mm} / \mathrm{s}$ were selected. Regarding a focal spot diameter of $2 w_{0}=300 \mu \mathrm{m}$, the radiant exposure was approximately $\Phi=6 \mathrm{~J} / \mathrm{cm}^{2}$. The irradiated area was moistened with water after every second area processing step to avoid thermal damage. In total, the scan trajectories were applied repeatedly for ten times (depth map) and thirty times (tissue thickness) respectively to visualize the boundaries in OCT imaging.

\subsection{Image processing}

The workflow of our implemented image processing consists of four general steps: acquisition, preprocessing, segmentation, and surface reconstruction. The OCT is calibrated before application compensating for intrinsic image distortions.

\subsubsection{Acquisition}

For the acquisition of datasets, a Thorlabs Ganymede Spectral Domain OCT with $930 \mathrm{~nm}$ center wavelength was employed. For the presented example of depth map extraction, a spoke scan pattern was preferred over stacking patterns in order to reduce computational costs. After each scan, a rotation of $3^{\circ}$ is executed. A total of 60 B-scans (512 px lateral; 1024 px axial) was acquired using a scan width of $10 \mathrm{~mm}$, in accordance with a lateral resolution of $19 \mu \mathrm{m}$. The detection of subsequent boundaries was evaluated on single B-scans (512 px lateral; 1024 px axial) with $2 \mathrm{~mm}$ scan width and a lateral resolution of $4 \mu \mathrm{m}$. In order to achieve precise measurements, the region of interest is reduced to the bottom of the ablation crater. The axial resolution was $2.7 \mu \mathrm{m}$ (in air) in both setups.

\subsubsection{Preprocessing}

Segmentation performance is mainly defined by the edge map obtained from the raw image. Since all acquired images are subject to noise and artifacts, a preprocessing step with speckle noise reduction is required to improve the edge map. A nonlinear anisotropic diffusion filter [14] is preferred over traditional Gaussian or mean filtering. In this case, the Perona-Malik model was implemented using a conduction coefficient of $\lambda=50$ and 20 iterations.

\subsubsection{Segmentation}

The presented work is based on traditional Kass snakes [15] which have been adapted to meet our requirements. A snake is a parametric curve $\vec{c}(s)=(x(s), \mathrm{y}(s))$ which is normalized to the arc length $s \in[0,1]$ within the image domain $I(x, y)$. The deformation and movement of the curve is driven by local minimization of an energy functional

$$
E_{\text {snake }}=\frac{1}{2} \int_{0}^{1} E_{\text {int }}+E_{\text {ext }} d s,
$$

which is composed of internal and external energies. Internal energy

$$
E_{\text {int }}=\alpha\left|\frac{\partial c(s)}{\partial s}\right|^{2}+\beta\left|\frac{\partial^{2} c(s)}{\partial s^{2}}\right|^{2}
$$

is equal to potential energy caused by tension and bending along the curve. This intrinsic condition is described physically as the sum of the square magnitudes of first and second order derivatives of the parametric curve, similar to a generalization of Hooke's law and elastic bending [11]. In contrast, external energy considers only image dependent properties. The traditional model sets

$$
E_{\text {ext }}=\gamma|\nabla I(x, y)|^{2}
$$

evaluating the contour $\vec{c}(s)$ on the squared magnitude gradient of the image $I(x, y)$. The individual contribution of the energy terms is adjusted and balanced by weighting factors $\alpha, \beta$ and $\gamma$.

The application of calculus of variations to the snake energy involves finding a minimum of the integral and leads to an Euler-Lagrange differential equation

$$
-\alpha \frac{\partial c(s)}{\partial s}+\beta \frac{\partial^{2} c(s)}{\partial s^{2}}+\gamma|\nabla I(x, y)|^{2}=0 .
$$

To overcome the poor abilities of outlining concave boundaries and to enlarge the capturing range of edges, we substitute the traditional formulation of gradient-based energies with a gradient vector flow (GVF) field $\vec{w}(x, y)$ [16] and artificial vertical pressure $\vec{n}$. Implicit Euler timestepping is applied to solve the dynamic system numerically and to compute snake evolution to a local equilibrium

$$
\frac{\partial c(s, t)}{\partial t}=-\alpha \frac{\partial c(s)}{\partial s}+\beta \frac{\partial^{2} c(s)}{\partial s^{2}}+\gamma \cdot \vec{w}(x, y)+\vec{n}
$$

Derivatives are approximated by central finite differences. After each step of evolution, boundary conditions con- 
strain the first and last contour point to vertical translation, and resampling of all contour points to equidistant spacing is applied for numerical reasons.

Before employing the snake algorithm, an initialization must be determined automatically. After capturing the raw image, anisotropic filtering reduces speckle noise. A fixed threshold of 0.5 is used to binarize the normalized image. Subsequently, the binary image is morphologically dilated using a 10 px by 10 px square structuring element to close holes and smooth boundaries. Binary labeling is used to detect connected components and eliminate isolated regions which do not comply with an area threshold. The initial contour is obtained from extracting and median filtering the specific boundary of the binary blob. After determination of the initial setup, the GVF field is calculated from the preprocessed raw image and the aforementioned snake algorithm is applied. So far, the proposed workflow uses unoptimized MATLAB code.

The weighting factors of the snake were set to $\alpha=4$, $\beta=0.5$, and $\gamma=4$. The GVF regularization factor was set to $\mu=0.1$ and 20 iterations were performed. The artificial pressure was set to $\vec{n}=(0, \pm 1 \mathrm{px})$ for each evolution. These parameters were determined empirically.

\subsubsection{Surface reconstruction}

The segmented contours $\vec{c}(s)$ are defined locally in the image domain $I(x, y)$ of each specific B-scan. In order to visualize and reconstruct the depth map from rotationally acquired datasets, a conversion between local and global coordinates was performed. Thus, local coordinates were transformed to a global Cartesian coordinate system and interpolated using cylindrical coordinates, based on the acquisition angle of each B-scan. Additionally, scaling was applied in accordance to the selected OCT settings.

\section{Results}

We present our experimental results to prove the suitability of the snake algorithm to outline surface and underlying anatomical boundaries. Regarding the selected laser settings, no charred tissue could be visually identified causing shadows or artifacts in the examined OCT datasets. Therefore, the system is applicable for gentle processing of biological hard tissue with minor thermal damage.

\subsection{Depth information}

For evaluation of our proposed workflow we use OCT images presenting the bone surface before and after laser ablation. The snake algorithm was performed on a specific set of 60 rotationally acquired OCT images for each condition. Image 2 shows the performance of the snake for an acquisition angle of $90^{\circ}$. In this case, the surface was outlined successfully by automatic initialization close to the boundary. Although the surface topography changes significantly within datasets, it should be pointed out that only few outliners occurred and only close to the image boundaries. Therefore, the region of interest (ablation ge- ometry) was not affected. After conversion from local to global coordinates, the surface reconstruction was applied.

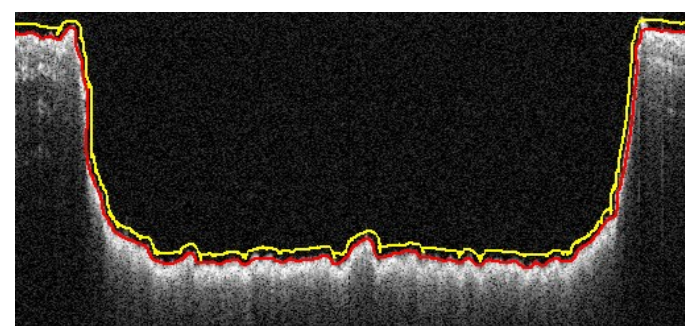

Image 2: Cropped OCT B-scan of bone surface with processed circular pattern ( $8 \mathrm{~mm}$ diameter) and segmentation Yellow and red lines indicate initialization of the snake and final boundary respectively.

The reconstructed surfaces of raw and processed bone are shown in image 3 (a) and (b) respectively. In order to determine the ablation depth exclusively, the difference of raw and processed surface was calculated. Therefore, the representation in image 3 (c) only contains depth information concerning the laser ablation. The depth chart in image 3 (d) displays a homogeneous ablation and clarifies the influence of tissue properties on laser processing.

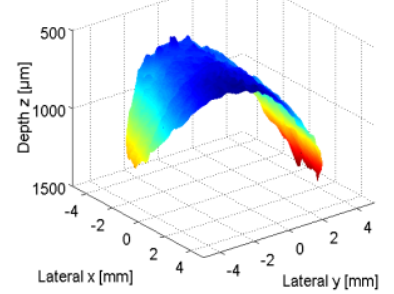

(a)

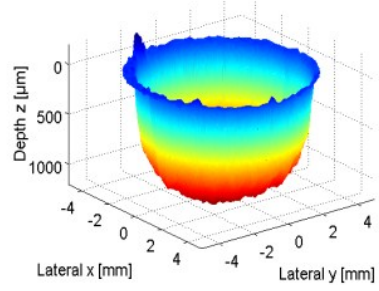

(c)

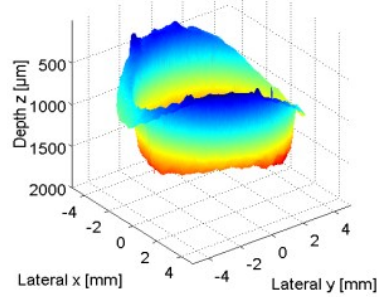

(b)

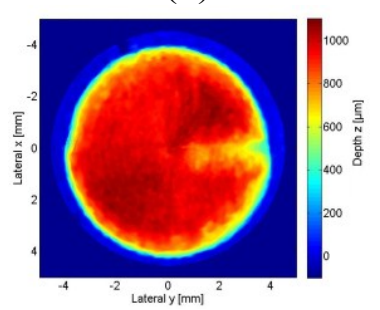

(d)
Image 3: (a) Reconstruction of raw bone surface. (b) Reconstruction of bone surface after laser ablation. (c) Difference of surfaces in (a) and (b). (d) Depth chart of circular ablation showing mostly uniform depth and a small ridge on the right due to inhomogeneous bone tissue.

\subsection{Second boundary detection}

For the purpose of protecting underlying anatomical structures, we evaluated the feasibility of automatic detection and quantitative thickness determination of remaining tissue using OCT imaging and snakes. Twice, the proposed snake algorithm was applied to the same B-scan displaying a specific section of the bottom of the ablation crater and underlying anatomical boundary. Image 4 (a) shows the detection performance with outlined boundaries. The thickness profile in image 4 (b) was calculated by undistortion of the vertical distance between upper and lower 
boundary and a refractive index of 1.55 for bone [17]. This enables to identify regions which should be excluded from further processing.

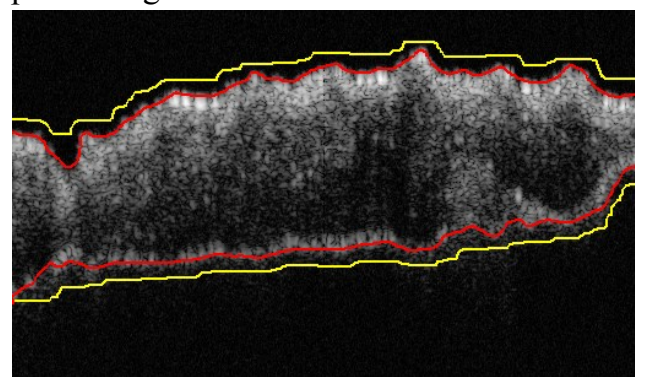

(a)

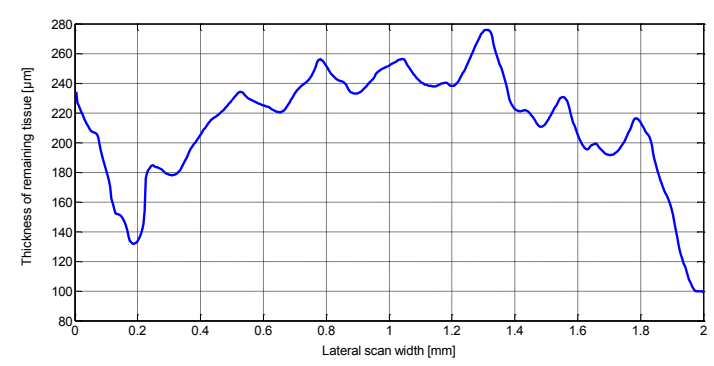

(b)

Image 4: (a) OCT image and segmentation of remaining tissue. Yellow and red lines indicate initialization of the snake and final contour respectively. (b) Thickness profile of the tissue layer

Penetration depth of OCT is strongly dependent on tissue water content. Dehydration caused by preceding laser ablation promotes strong scattering in the surface layer, resulting in decreased imaging depth. Therefore, a thin layer of water was applied. Scattering was observed only until the water was absorbed. Underlying concavities and boundaries became detectable. Thus, moistening is essential for successful imaging of subsurface structures in bone.

\section{Conclusion}

This paper demonstrates the feasibility of interactive depth measurements during laser tissue ablation. Robustness of our approach for surface topology segmentation using adapted snakes algorithm and $3 \mathrm{D}$ reconstruction is proven on laser ablated bone specimen. A depth chart is obtained as a prospective instrument for interactive ablation control. In order to improve process safety, we also present the means to determine remaining bone thickness by adapting our algorithm to identify a second tissue boundary in OCT imaging. Future research will focus on minimizing computational time necessary to extract ablation depth information as well as on the implementation of adaptive algorithms to automate parameterization.

\section{$5 \quad$ References}

[1] S. Stopp, D. Svejdar, E. von Kienlin, H. Deppe, and T. Lueth, "A new approach for creating defined geometries by navigated laser ablation based on volu- metric 3-d data," Trans. Bio. Eng., vol. 55, no. 7, pp. 1872-1880, 2008.

[2] L. A. Kahrs, "Bildverarbeitungsunterstützte Laserknochenablation am humanen Felsenbein," dissertation, Universität Fridericiana zu Karlsruhe, 2009.

[3] A. Vogel and V. Venugopalan, "Mechanisms of pulsed laser ablation of biological tissues," Chem. Rev., vol. 103, pp. 577-644, 2003.

[4] J. Walsh, T. J. Flotte, and T. F. Deutsch, "Er:yag laser ablation of tissue: Effect of pulse duration and tissue type on thermal damage," Las. Surg. Med., vol. 9, pp. 314-326, 1989.

[5] J. S. Nelson, A. Orenstein, L.-H. L. Liaw, and M. W. Berns, "Mid-infrared erbium:yag laser ablation of bone: The effect of laser osteotomy on bone healing," Las. Surg. Med., vol. 9, pp. 362-374, 1989.

[6] M. H. Niemz, Laser-Tissue Interactions. Springer Berlin Heidelberg, 2007.

[7] U. Romeo, A. Del Vecchio, G. Palaia, G. Tenore, P. Visca, and C. Maggiore, "Bone damage induced by different cutting instruments - an in vitro study," Braz. Dent. J., vol. 20, pp. 162-168, 2009.

[8] A. Murtaza and P. Renuka, "Signal processing overview of optical coherence tomography for medical imaging," Texas Instrumens White Paper, 2010.

[9] W. Drexler and J. G. Fujimoto, Eds., Optical Coherence Tomography Technology and Applications. Springer Berlin Heidelberg, 2008.

[10] O. Müller, S. Donner, T. Klinder, R. Dragon, I. Bartsch, F. Witte, A. Krüger, A. Heisterkamp, and B. Rosenhahn, "Model based 3d segmentation and oct image undistortion of percutaneous implants," MICCAI, vol. III, pp. 454-462, 2011.

[11] D. Fernandez, "Delineating fluid-filled region boundaries in optical coherence tomography images of the retina," Medical Imaging, IEEE Transactions on, vol. 24, no. 8, pp. 929-945, 2005.

[12] S. A. Boppart, J. Herrmann, and C. Pitris, "Highresolution optical coherence tomography-guided laser ablation of surgical tissue," J. Surg. Res., vol. 82, pp. 275-284, 1999.

[13] M. Ohmi, M. Ohnishi, D. Takada, and M. Haruna, "Real-time oct imaging of laser ablation of biological tissue," vol. 7562, no. 1. SPIE, 2010, p. 756210.

[14] P. Perona and J. Malik, "Scale-space and edge detection using anisotropic diffusion," IEEE Trans. Pattern Anal. Mach. Intell., vol. 12, no. 7, pp. 629-639, 1990.

[15] M. Kass, A. Witkin, and D. Terzopoulos, "Snakes: Active contour models," Int. J. Comp. Vis., vol. 1, pp. 321-331, 1988.

[16] C. Xu and J. L. Prince, "Generalized gradient vector flow external forces for active contours," Signal Processing, vol. 71, no. 2, pp. 131 - 139, 1998.

[17] A. Ascenzi and C. Fabry, "Technique for dissection and measurement of refractive index of osteones," $J$ Biophys Biochem Cytol, vol. 6, pp. 139-142, 1959. 